\title{
Uniformly Convergent Computational Technique for Singularly Perturbed Self-adjoint Mixed Boundary-Value Problems
}

\author{
Rajesh K. Bawa ${ }^{1}$ and S. Natesan ${ }^{2}$ \\ 1 Department of Computer Science, Punjabi University, \\ Patiala - 147 002, India \\ 2 Department of Mathematics, Indian Institute of Technology, \\ Guwahati - 781 039, India
}

\begin{abstract}
In this paper, we propose a second-order parameter-uniform convergent hybrid scheme for self-adjoint singular perturbation problems (SPPs) subject to mixed (Robin) type conditions. The cubic spline baesd difference scheme is combined with the classical central difference scheme to obtain monotone scheme. Numerical example is provided to support the theory.
\end{abstract}

Keywords: Finite difference scheme, cubic splines, singular perturbation problems, piece-wise uniform meshes.

Subject Classification: AMS 65L10 CR G1.7.

\section{Introduction}

Singular perturbation problems (SPPs) arise in several branches of engineering and applied mathematics which include fluid dynamics, quantum mechanics, elasticity, chemical reactor theory, gas porous electrodes theory, etc. To solve these types of problems various methods are proposed in the literature, more details can be found in the books of Farrell et al. [2], and Roos et al. 6].

We consider the following singularly perturbed self-adjoint boundary-value problem (BVP):

$$
\begin{gathered}
L u(x) \equiv-\varepsilon u^{\prime \prime}(x)+b(x) u(x)=f(x), \quad x \in D=(0,1) \\
\alpha_{1} u(0)-\beta_{1} u^{\prime}(0)=A, \quad \alpha_{2} u(1)+\beta_{2} u^{\prime}(1)=B,
\end{gathered}
$$

where $\alpha_{1}, \beta_{1}, \alpha_{2}, \beta_{2}>0$ and $\varepsilon>0$ is a small parameter, $b$ and $f$ are sufficiently smooth functions, such that $b(x) \geq \beta>0$ on $\bar{D}=[0,1]$. Under these assumptions, the BVP (1/2) possesses a unique solution $u(x) \in C^{2}(D) \cap C^{1}(\bar{D})$. In general, the solution $u(x)$ may exhibit two boundary layers of exponential type at both end points $x=0,1$. Boundary-value problems of the type (1, 2) arise in many applications, for instance, confinement of a plasma column by reaction pressure, theory of gas porous electrodes, performance of catalytic pellets and geophyisical fluid dynamics chemical reactions [1,5]. In [3], the authors 
have devised HODIE schemes for singularly perturbed convection-diffusion and reaction-diffusion problems respectively.

For sufficiently small $\varepsilon$, classical methods on uniform meshes only work for very large number of mesh points. Nevertheless, if these methods are defined on special fitted meshes, the convergence to the exact solution is uniform in $\varepsilon$. Shishkin meshes are simple piecewise uniform meshes of this kind, frequently used for singularly perturbed problems. For above mentioned problem, The Shishkin mesh $\bar{\Omega}$ is constructed as follows. The domain $\bar{\Omega}$ is divided into three subintervals as $\bar{\Omega}=[0, \sigma] \cup[\sigma, 1-\sigma] \cup[1-\sigma, 1]$ for some $\sigma$ such that $0<\sigma \leq 1 / 4$. On the subintervals $[0, \sigma],[1-\sigma, 1]$ a uniform mesh with $N / 4$ mesh-intervals is placed, where $[\sigma, 1-\sigma]$ has a uniform mesh with $N / 2$ mesh intervals. It is obvious that the mesh is uniform when $\sigma=1 / 4$, and it is fitted to the problem by choosing $\sigma=\min \left\{\frac{1}{4}, \sigma_{0} \sqrt{\varepsilon} \ln N\right\}$, where $\sigma_{0}$ is a constant will be fixed later. Further, we denote the mesh size in the regions $[\sigma, 1-\sigma]$ as $h^{(1)}=2(1-2 \sigma) / N$, and in $[0, \sigma],[1-\sigma, 1]$ by $h^{(2)}=4 \sigma / N$. Here, we propose an hybrid scheme which is a mixture of the cubic spline scheme with the classical central difference scheme for the BVP (1-2) on above mentioned Shishkin mesh. We apply the cubic spline difference scheme in the inner region $(0, \sigma) \cup(1-\sigma, 1)$, whereas in the outer region $(\sigma, 1-\sigma)$ we use the classical central difference scheme. This is mainly because to retain the discrete maximum principle of the difference scheme. The present method provides second-order uniform convergence throughout the domain of interest. A numerical experiment have been carried out to show the efficiency of the method.

\section{$2 \varepsilon$-Uniform Hybrid Scheme}

The cubic spline based scheme is analyzed for stability and convergence and it is observed that for the corresponding matrix to be a M-matrix, a very restrictive condition is needed on the mesh size, specially in the outer region where a coarse mesh is enough to reflect the behavior of the solution in that region. So, to overcome this, The following hybrid scheme is proposed in which the well known classical central difference scheme is taken in the outer region and the cubic spline scheme in boundary layer region

$$
r_{i}^{-} u_{i-1}+r_{i}^{c} u_{i}+r_{i}^{+} u_{i+1}=q_{i}^{-} f_{i-1}+q_{i}^{c} f_{i}+q_{i}^{+} f_{i+1}, \quad i=1, \cdots, N-1,
$$

along with following equations for approximations at boundaries

$$
\left\{\begin{array}{l}
r_{0}^{c} u_{0}+r_{0}^{+} u_{1}=q_{0}^{-}+q_{0}^{c} f_{0}+q_{0}^{+} f_{1}, \\
r_{N}^{-} u_{N-1}+r_{N}^{c} u_{0}=q_{N}^{-}+q_{N}^{c} f_{N-1}+q_{N}^{+} f_{N},
\end{array}\right.
$$

for $i=1, \cdots, N / 4$ and $3 N / 4, \cdots, N-1$

$$
\left\{\begin{array}{l}
r_{i}^{-}=\frac{-3 \varepsilon}{h_{i-1}\left(h_{i}+h_{i-1}\right)}+\frac{h_{i-1}}{2\left(h_{i}+h_{i-1}\right)} b_{i-1} ; \quad r_{i}^{c}=\frac{3 \varepsilon}{h_{i} h_{i-1}}+b_{i} ; \\
r_{i}^{+}=\frac{-3 \varepsilon}{h_{i}\left(h_{i}+h_{i-1}\right)}+\frac{h_{i}}{2\left(h_{i}+h_{i-1}\right)} b_{i+1} ;
\end{array}\right.
$$




$$
\left\{q_{i}^{-}=\frac{h_{i-1}}{2\left(h_{i}+h_{i-1}\right)} ; \quad q_{i}^{c}=1 ; \quad q_{i}^{+}=\frac{h_{i}}{2\left(h_{i}+h_{i-1}\right)},\right.
$$

and for $i=N / 4+1, \cdots, 3 N / 4-1$

$$
\begin{gathered}
\left\{r_{i}^{-}=\frac{-2 \varepsilon}{h_{i-1}\left(h_{i}+h_{i-1}\right)} ; \quad r_{i}^{c}=\frac{2 \varepsilon}{h_{i} h_{i-1}}+b_{i} ; \quad r_{i}^{+}=\frac{-2 \varepsilon}{h_{i}\left(h_{i}+h_{i-1}\right)},\right. \\
\left\{q_{i}^{-}=0 ; \quad q_{i}^{c}=1 ; \quad q_{i}^{+}=0 .\right.
\end{gathered}
$$

and

$$
\left\{\begin{array}{l}
r_{0}^{c}=-\frac{3 \varepsilon}{h_{0}}\left(\alpha_{1}+\frac{\beta_{1}}{h_{0}}\right)-b_{0} \beta_{1} ; \quad r_{0}^{+}=-\frac{3 \varepsilon \beta_{1}}{h_{0}^{2}}+\frac{b_{1}}{2} \beta_{1} \\
q_{0}^{-}=-\frac{3 \varepsilon A}{h_{0}} ; \quad q_{0}^{c}=-\beta_{1} ; \quad q_{0}^{+}=-\frac{\beta_{1}}{2} \\
r_{N}^{-}=-\frac{3 \varepsilon \beta_{2}}{h_{N-1}^{2}}+\frac{b_{N-1}}{2} \beta_{2} ; \quad r_{N}^{c}=-\frac{3 \varepsilon}{h_{N-1}}\left(\alpha_{2}+\frac{\beta_{2}}{h_{N-1}}\right)-\frac{b_{N}}{2} \beta_{2} \\
q_{N}^{-}=-\frac{3 \varepsilon B}{h_{N-1}} ; \quad q_{N}^{c}=-\frac{\beta_{2}}{2} ; \quad q_{N}^{+}=-\beta_{2} .
\end{array}\right.
$$

\section{$3 \quad$ Numerical Experiments}

To show the accuracy of the present method, here we have implemented it to a test problem. The results are presented in the form of tables with maximum point-wise errors and rate of convergent. Table 1 display the results for the

\begin{tabular}{|c|c|c|c|c|c|c|c|}
\hline$\varepsilon$ & \multicolumn{7}{|c|}{ Number of mesh points $N$} \\
\hline & 16 & 32 & 64 & 128 & 256 & 512 & 1024 \\
\hline \multirow[t]{2}{*}{$2^{-4}$} & $2.0176 \mathrm{e}-2$ & $4.9167 \mathrm{e}-3$ & $1.2214 \mathrm{e}-3$ & $3.0487 \mathrm{e}-4$ & $7.6188 \mathrm{e}-5$ & $1.9045 \mathrm{e}-5$ & $4.7611 \mathrm{e}-6$ \\
\hline & 2.0369 & 2.0092 & 2.0023 & 2.0006 & 2.0001 & 2.0000 & \\
\hline \multirow[t]{2}{*}{$2^{-16}$} & $1.5583 \mathrm{e}-1$ & $5.6409 \mathrm{e}-2$ & $1.9283 \mathrm{e}-2$ & $6.4067 \mathrm{e}-3$ & $2.0840 \mathrm{e}-3$ & $6.5813 \mathrm{e}-4$ & $2.0297 \mathrm{e}-5$ \\
\hline & 1.4660 & 1.5486 & 1.5897 & 1.6203 & 1.6629 & 1.6971 & \\
\hline \multirow[t]{2}{*}{$2^{-24}$} & $1.5515 \mathrm{e}-1$ & $5.6201 \mathrm{e}-2$ & $1.9217 \mathrm{e}-2$ & $6.3854 \mathrm{e}-3$ & $2.0771 \mathrm{e}-3$ & $6.5597 \mathrm{e}-4$ & $2.0231 \mathrm{e}-5$ \\
\hline & 1.4650 & 1.5482 & 1.5895 & 1.6202 & 1.6629 & 1.6971 & \\
\hline \multirow[t]{2}{*}{$2^{-32}$} & $1.5512 \mathrm{e}-1$ & $5.6192 \mathrm{e}-2$ & $1.9214 \mathrm{e}-2$ & $6.3844 \mathrm{e}-3$ & $2.0768 \mathrm{e}-3$ & $6.5588 \mathrm{e}-4$ & $8 \mathrm{e}-5$ \\
\hline & 1.4649 & 1.5482 & 1.5895 & 1.6202 & 1.6629 & 1.6971 & \\
\hline \multirow[t]{2}{*}{$2^{-36}$} & $1.5512 \mathrm{e}-1$ & $5.6191 \mathrm{e}-2$ & $1.9214 \mathrm{e}-2$ & $6.3844 \mathrm{e}-3$ & $2.0768 \mathrm{e}-3$ & $6.5587 \mathrm{e}-4$ & $2.0228 \mathrm{e}-5$ \\
\hline & 1.4649 & 1.5482 & 1.5895 & 1.6202 & 1.6629 & 1.6971 & \\
\hline \multirow[t]{2}{*}{$2^{-40}$} & $1.5512 \mathrm{e}-1$ & $5.6191 \mathrm{e}-2$ & $1.9214 \mathrm{e}-2$ & $6.3844 \mathrm{e}-3$ & $2.0768 \mathrm{e}-3$ & $6.5587 \mathrm{e}-4$ & $2.0228 \mathrm{e}-5$ \\
\hline & 1.4649 & 1.5482 & 1.5895 & 1.6202 & 1.6629 & 1.6971 & \\
\hline$G^{N}$ & $1.6274 \mathrm{e}-1$ & $5.8585 \mathrm{e}-2$ & $1.9599 \mathrm{e}-2$ & $6.5096 \mathrm{e}-3$ & $2.1171 \mathrm{e}-3$ & $6.6858 \mathrm{e}-4$ & $2.0619 \mathrm{e}-4$ \\
\hline$p_{\text {uni }}$ & 1.4740 & 1.5798 & 1.5902 & 1.6205 & 1.6630 & 1.6971 & \\
\hline
\end{tabular}
values $\varepsilon=2^{-4}, 2^{-16}, \cdots, 2^{-40}$ and different values of $N$.

Table 1. Maximum pointwise errors $G_{\varepsilon}^{N}$, rates of convergence p and $\varepsilon$ - uniform errors $G^{N}$ corresponding to the Hybrid scheme for Example 1 
Example 1. 7] Consider the self-adjoint SPP

$$
\begin{gathered}
-\varepsilon u^{\prime \prime}(x)+(1+x)^{2} u(x)=\left[4 x^{2}-14 x+4\right](1+x)^{2}, x \in(0,1) \\
u(0)-u^{\prime}(0)=0, \quad u(1)=0 .
\end{gathered}
$$

We use the following double mesh principle to calculate the maximum pointwise error and rate of convergence.

Let $\bar{D}_{\varepsilon}^{N}$ be a Shishkin mesh with the parameter $\sigma$ altered slightly to $\bar{\sigma}=$ $\min \left\{\frac{1}{4}, \sigma_{0} \sqrt{\varepsilon} \ln (N / 2)\right\}$, Then, for $i=0,1, \cdots N$, the $i$ th point of the mesh $\bar{D}_{\varepsilon}^{N}$ coincides with the (2i) the point of the mesh $\bar{D}_{\varepsilon}^{2 N}$. The double mesh difference is defined as $G_{\varepsilon}^{N}=\max _{x_{i} \in \bar{D}_{\varepsilon}^{N}}\left|U^{N}\left(x_{j}\right)-U^{2 N}\left(x_{j}\right)\right|, \quad$ and $\quad G^{N}=\max _{\varepsilon} G_{\varepsilon}^{N}$, where $U^{N}\left(x_{j}\right)$ and $U^{2 N}\left(x_{j}\right)$ respectively denote the numerical solutions obtained using $N$ and $2 N$ mesh intervals. Further, we calculate the parameter-robust orders of convergence as $p=\log _{2}\left(\frac{G_{\varepsilon}^{N}}{G_{\varepsilon}^{2 N}}\right)$ and $p_{\text {uni }}=\log _{2}\left(\frac{G^{N}}{G^{2 N}}\right)$. Here, we took $\sigma_{0}=1$. We have tabulated the results in Tables 1 which shows the maximum point-wise error and the rate of convergence for Example 1

\section{Conclusions}

In this paper, We have proposed a hybrid method for the numerical solution of singularly perturbed reaction-diffusion problems. The underlying idea of the method combines both the cubic spline and classical central difference scheme. The method is of second-order convergent. One test example is studied to verify the efficiency and accuracy of the theoretical error estimates, and they reflect perfectly the same.

\section{References}

1. R.C.Y. Chin and R.Krasny. A hybrid asymptotic finite-element method for stiff twopoint boundary-value problems. SIAM J. Sci. and Stat. Comput., 4:229-243,1983.

2. P.A. Farrell, A.F. Hegarty, J.J.H. Miller, E. O'Riordan, and G.I. Shishkin. Robust Computational Techniques for Boundary Layers. Chapman \& Hall/CRC Press, 2000 .

3. J.L. Gracia, F. Lisbona, and C. Clavero. High order $\varepsilon$-uniform methods for singularly perturbed reaction-diffusion problems. Lecture Notes in Computer Science, 1998:350-358, 2001.

4. J.J.H. Miller, E. O'Riordan, and G.I. Shishkin. Fitted Numerical Methods for Singular Perturbation Problems. World Scientific, Singapore, 1996.

5. C.E.Pearson. On a differential equation of boundary layer type. J. Math. Phys., 47(144):134-154,1968.

6. H.-G. Roos, M. Stynes, and L. Tobiska. Numerical Methods for Singularly Perturbed Differential Equations. Springer, Berlin, 1996.

7. M.Stojanovic. Numerical solution of initial and singularly perturbed two-point boundary value problems using adaptive spline function approximation. Publications de L'institut Mathematique., 43(57):155-163,1988. 